Supporting information

In Situ Synthesis of Amino Acid Functionalized Carbon Dots with Tunable Properties and their Biological Applications

\author{
Subrata Pandit, Pradipta Behera, Jagabandhu Sahoo and Mrinmoy De* \\ Department of Organic Chemistry, Indian Institute of Science, Bangalore-560094, India \\ Email: $\underline{m d @ i i s c . a c . i n}$
}


Transmission Electron Microscopy (TEM):
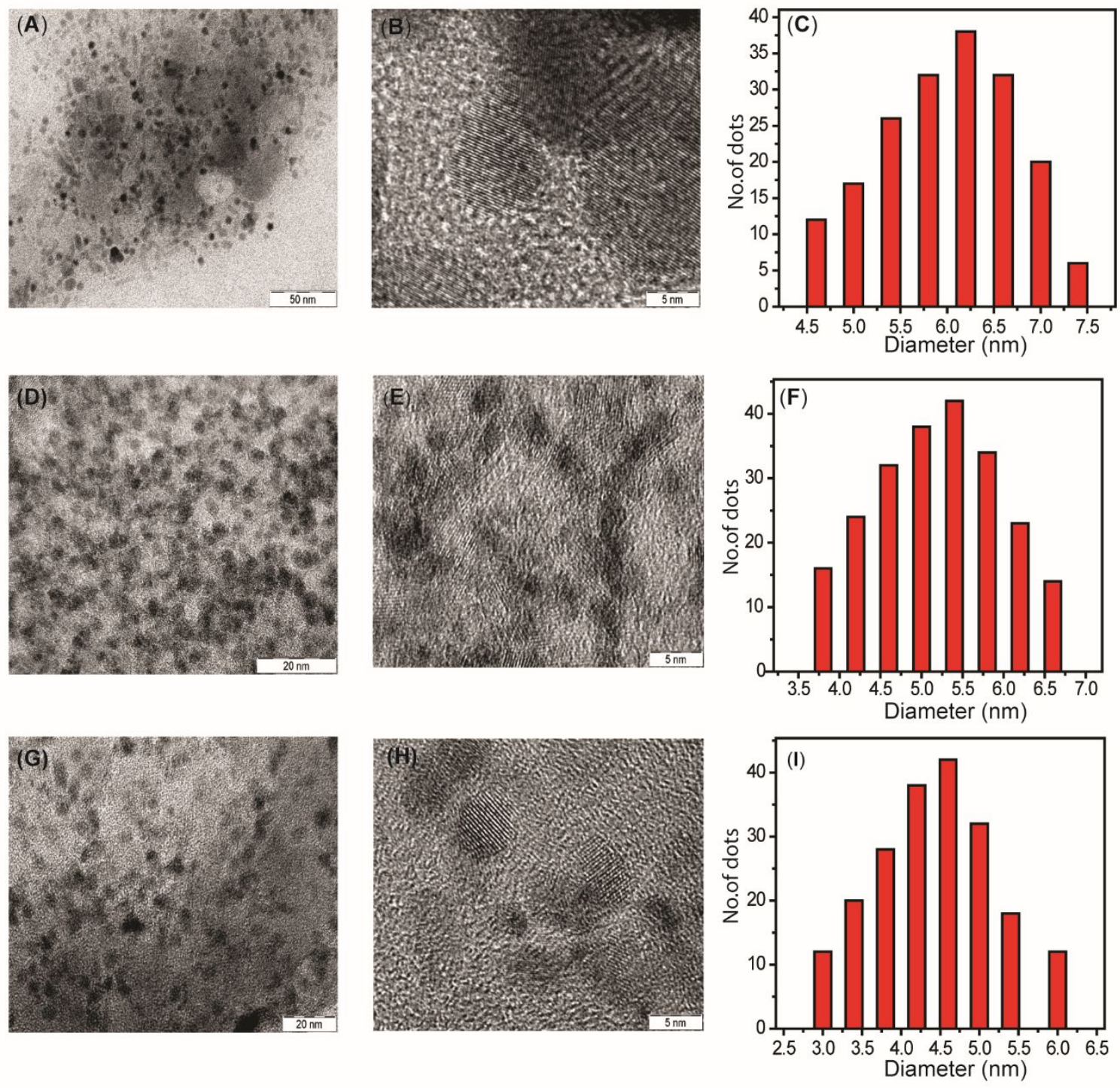

Figure S1. Transmission Electron Microscopy (TEM) image of amino acid functionalized carbon dots Phe C-Dots (A), His C-Dots (D) and Tyr C-Dots(G), HRTEM image of (B), (E) and (H), also size distribution plot of $(\mathrm{C}),(\mathrm{F})$ and $(\mathrm{I})$ respective amino acids 

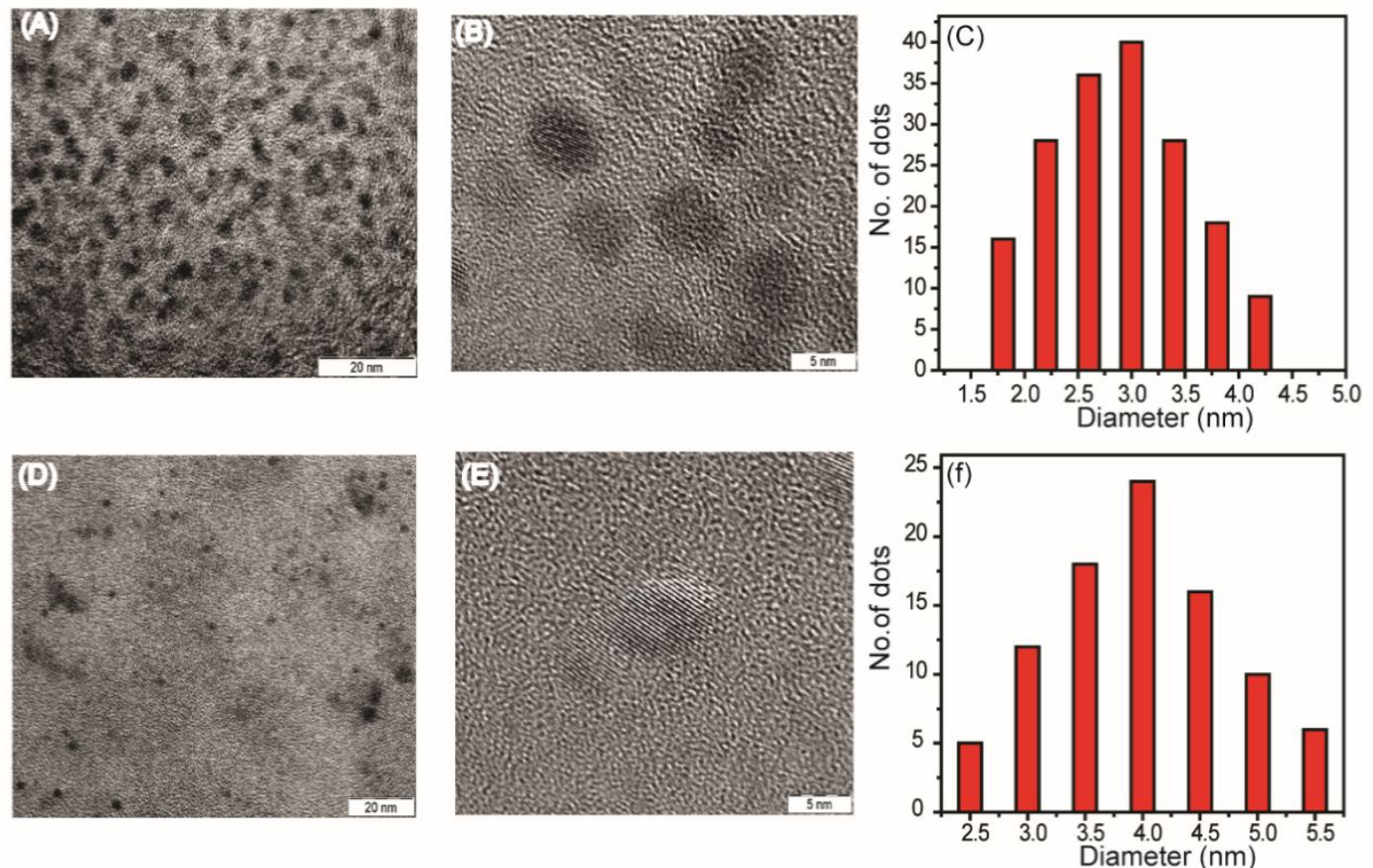

Figure S2. Transmission Electron Microscopy (TEM) image of amino acid functionalized carbon dots Arg C-Dots (A) and Cys C-Dots (D), HRTEM image of (B) and (E) also size distribution plot of $(\mathrm{C})$ and $(\mathrm{F})$ respective amino acids.

NMR Spectrum:
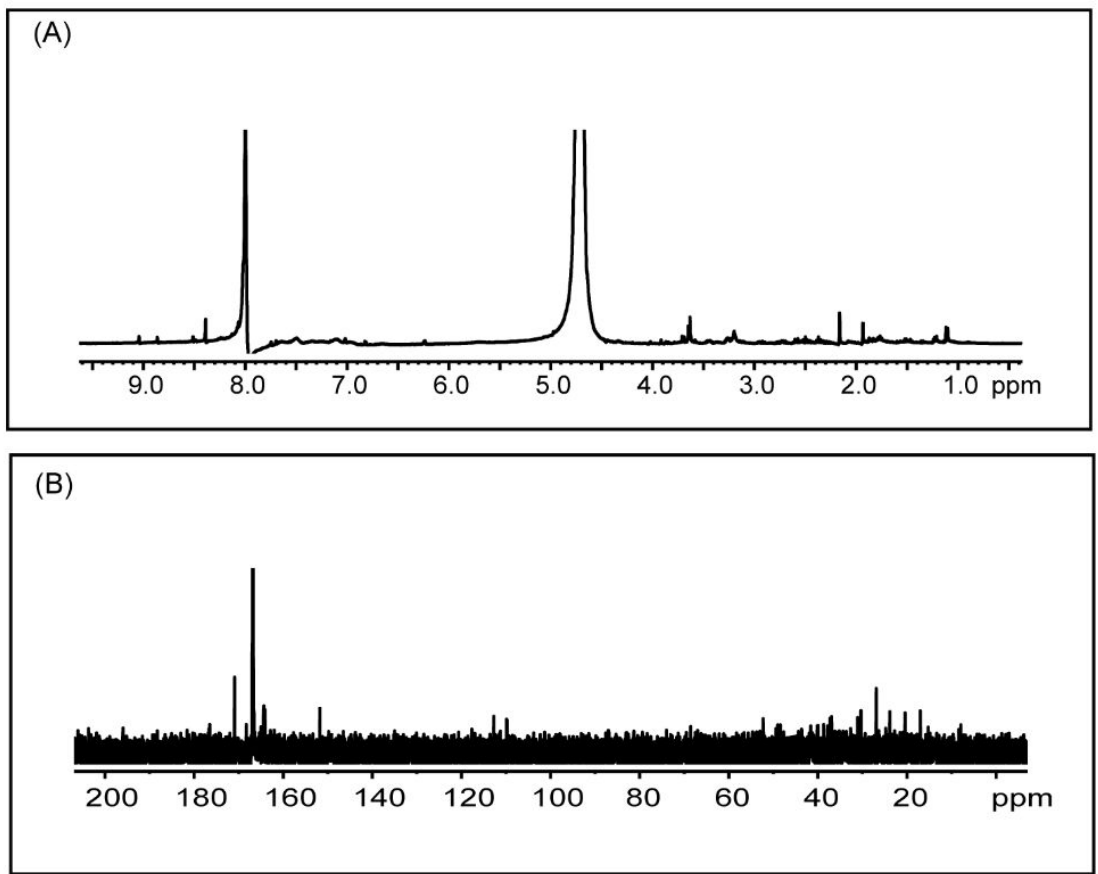

Figure S3. Nuclear Magnetic Resonance (NMR) Spectrum of Arg C-Dots (A) ${ }^{1} \mathrm{H}$ and (B) ${ }^{13} \mathrm{C}$ in $\mathrm{D}_{2} \mathrm{O}$ solvent (overnight scan) 

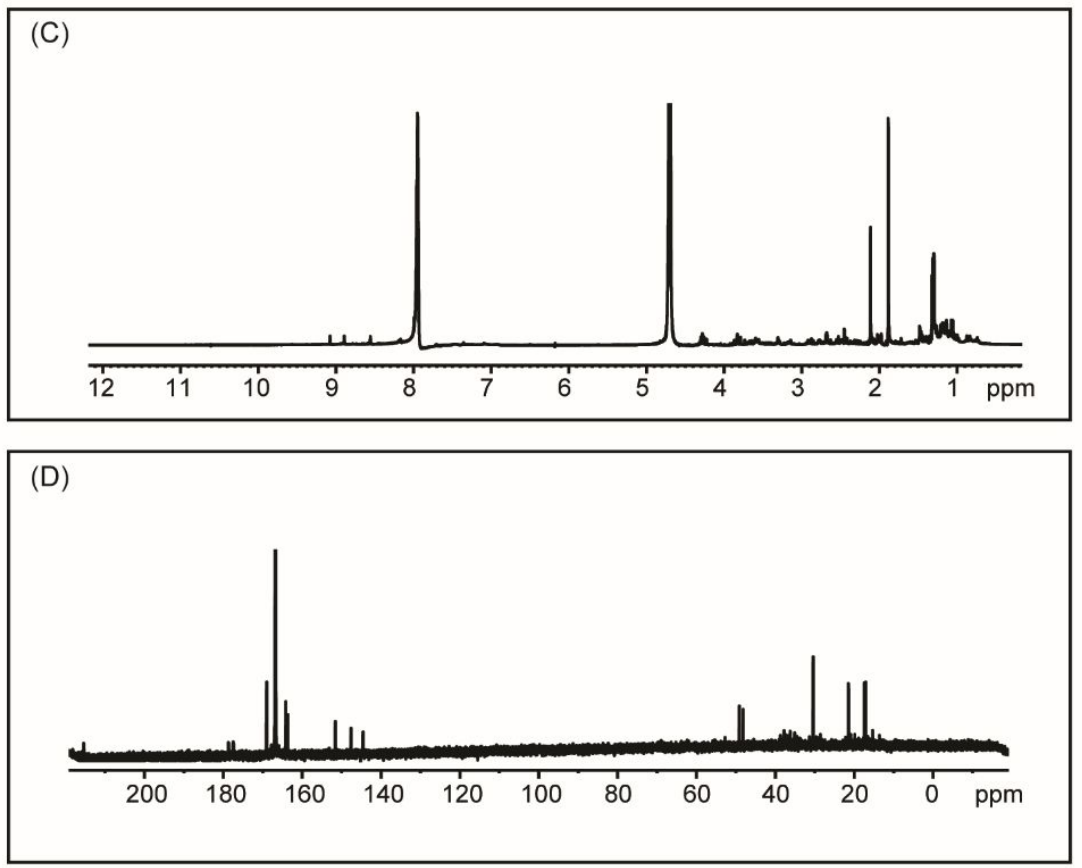

Figure S4. Nuclear Magnetic Resonance (NMR) Spectrum of Cys C-Dots (C) ${ }^{1} \mathrm{H}$ and (D) ${ }^{13} \mathrm{C}$ in $\mathrm{D}_{2} \mathrm{O}$ solvent (overnight scan)
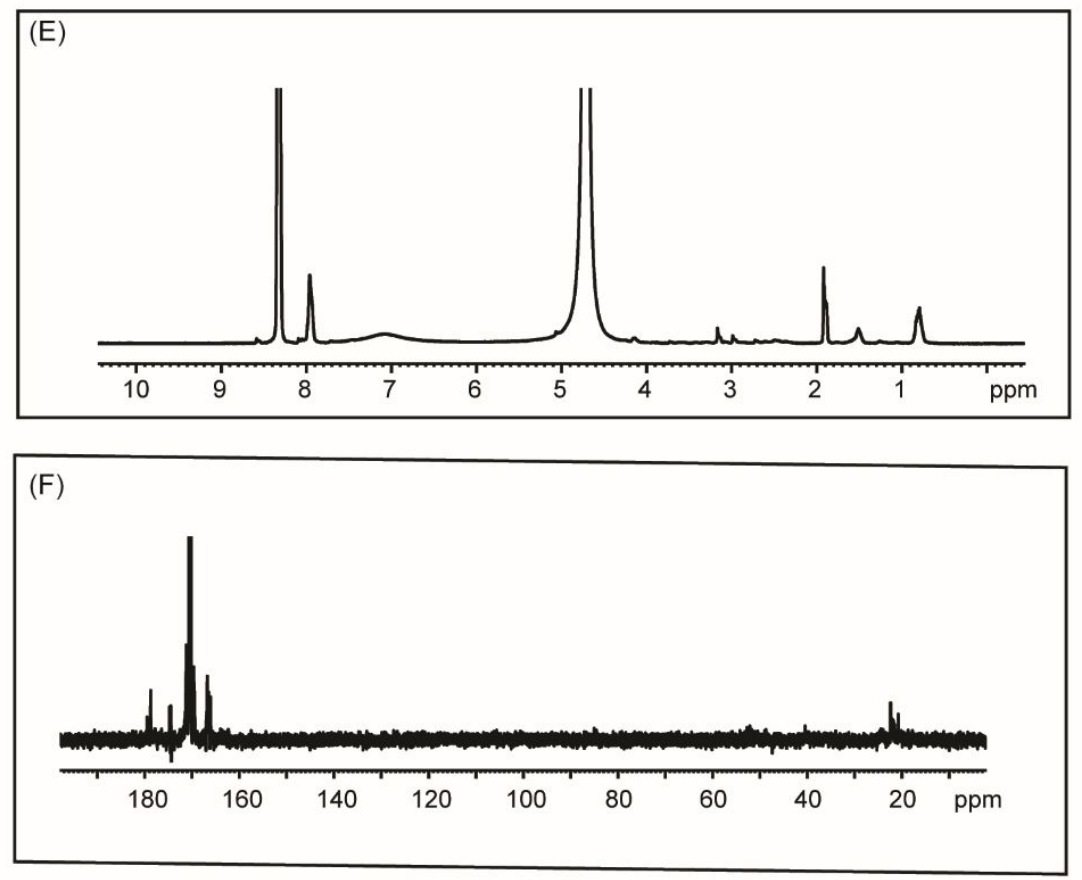

Figure S5. Nuclear Magnetic Resonance (NMR) Spectrum of Leu C-Dots $(\mathrm{E}){ }^{1} \mathrm{H}$ and $(\mathrm{F}){ }^{13} \mathrm{C}$ in $\mathrm{D}_{2} \mathrm{O}$ solvent (overnight scan) 

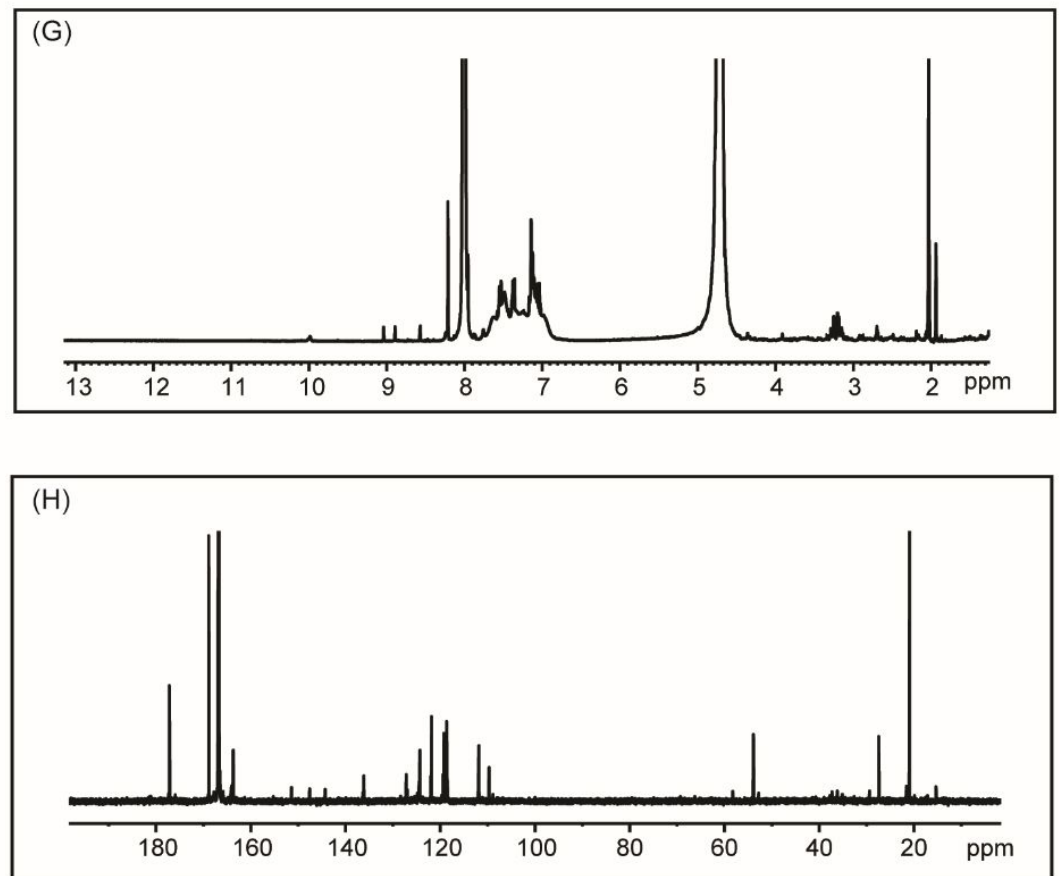

Figure S6. Nuclear Magnetic Resonance (NMR) Spectrum of $\operatorname{Trp}$ C-Dots $(\mathrm{G}){ }^{1} \mathrm{H}$ and $(\mathrm{H}){ }^{13} \mathrm{C}$ in $\mathrm{D}_{2} \mathrm{O}$ solvent (overnight scan)
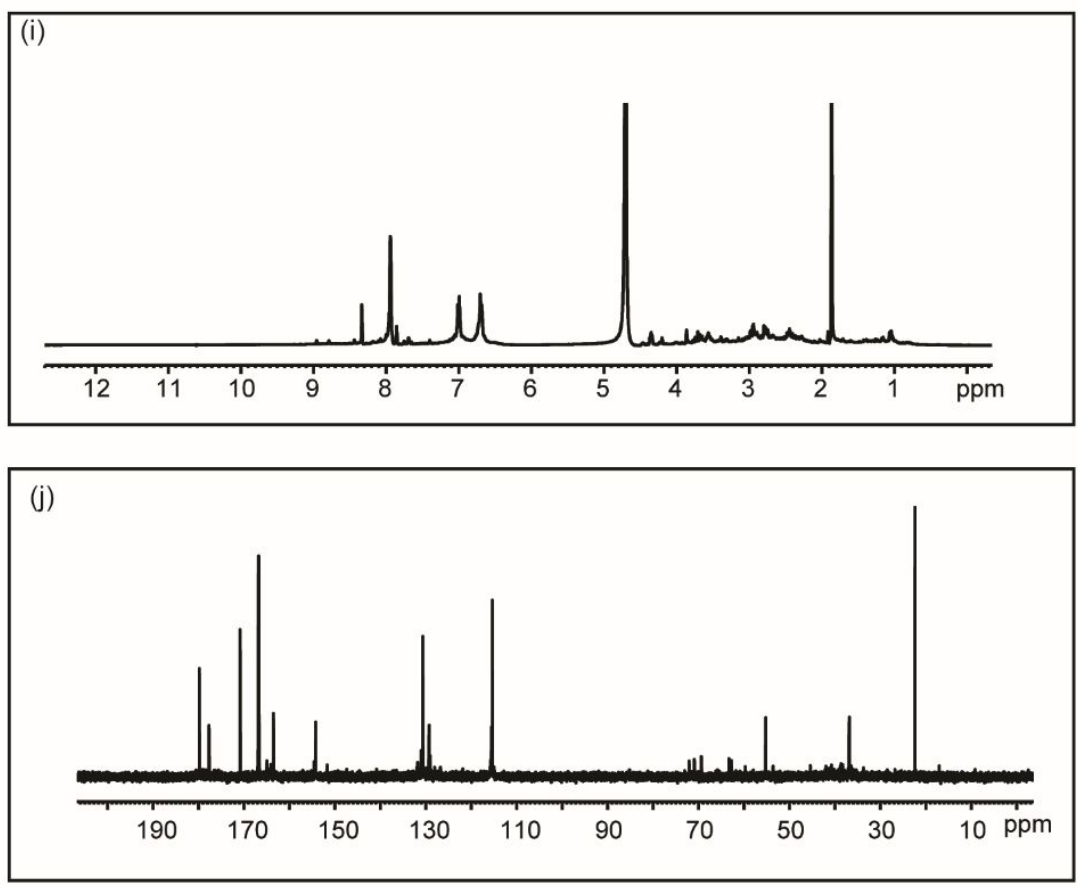

Figure S7. Nuclear Magnetic Resonance (NMR) Spectrum of Tyr C-Dots (I) ${ }^{1} \mathrm{H}$ and $(\mathrm{J}){ }^{13} \mathrm{C}$ in $\mathrm{D}_{2} \mathrm{O}$ solvent (overnight scan) 

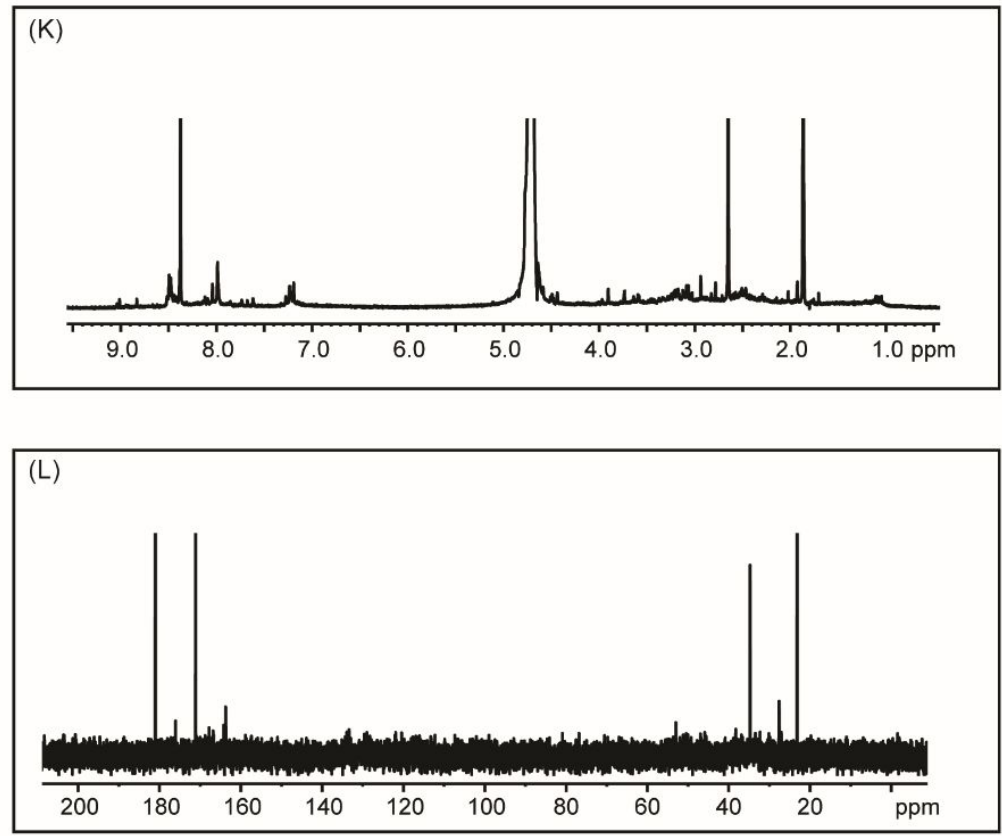

Figure S8. Nuclear Magnetic Resonance (NMR) Spectrum of His C-Dots $(\mathrm{K}){ }^{1} \mathrm{H}$ and $(\mathrm{L}){ }^{13} \mathrm{C}$ in $\mathrm{D}_{2} \mathrm{O}$ solvent (overnight scan)
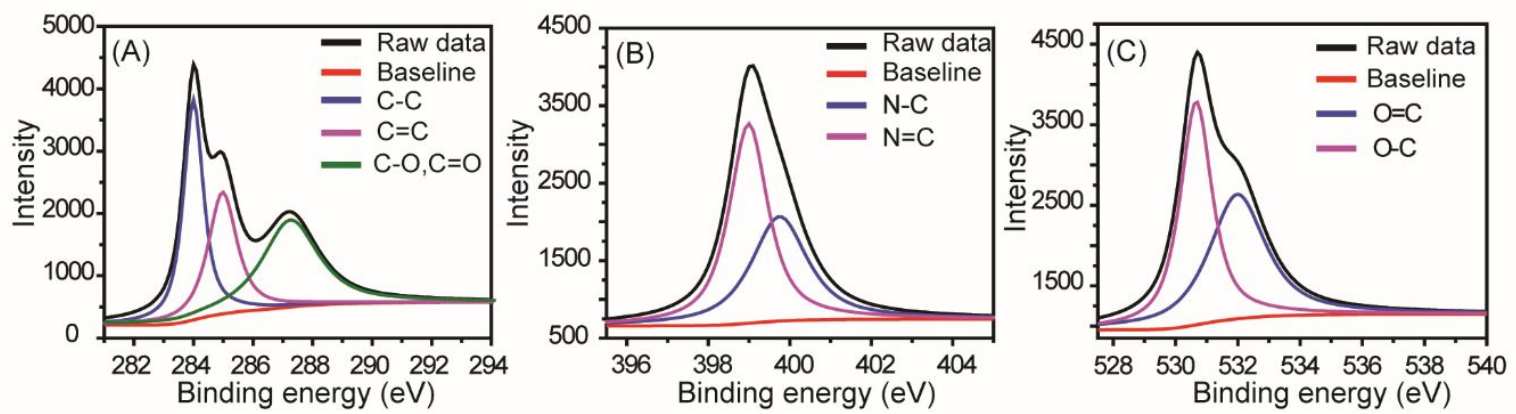
Figure S9. High resolution deconvoluting XPS Spectrum (A) C1s (B) N1s and (C) O1s of Arg CDots with black line (Raw data) and red line (baseline).
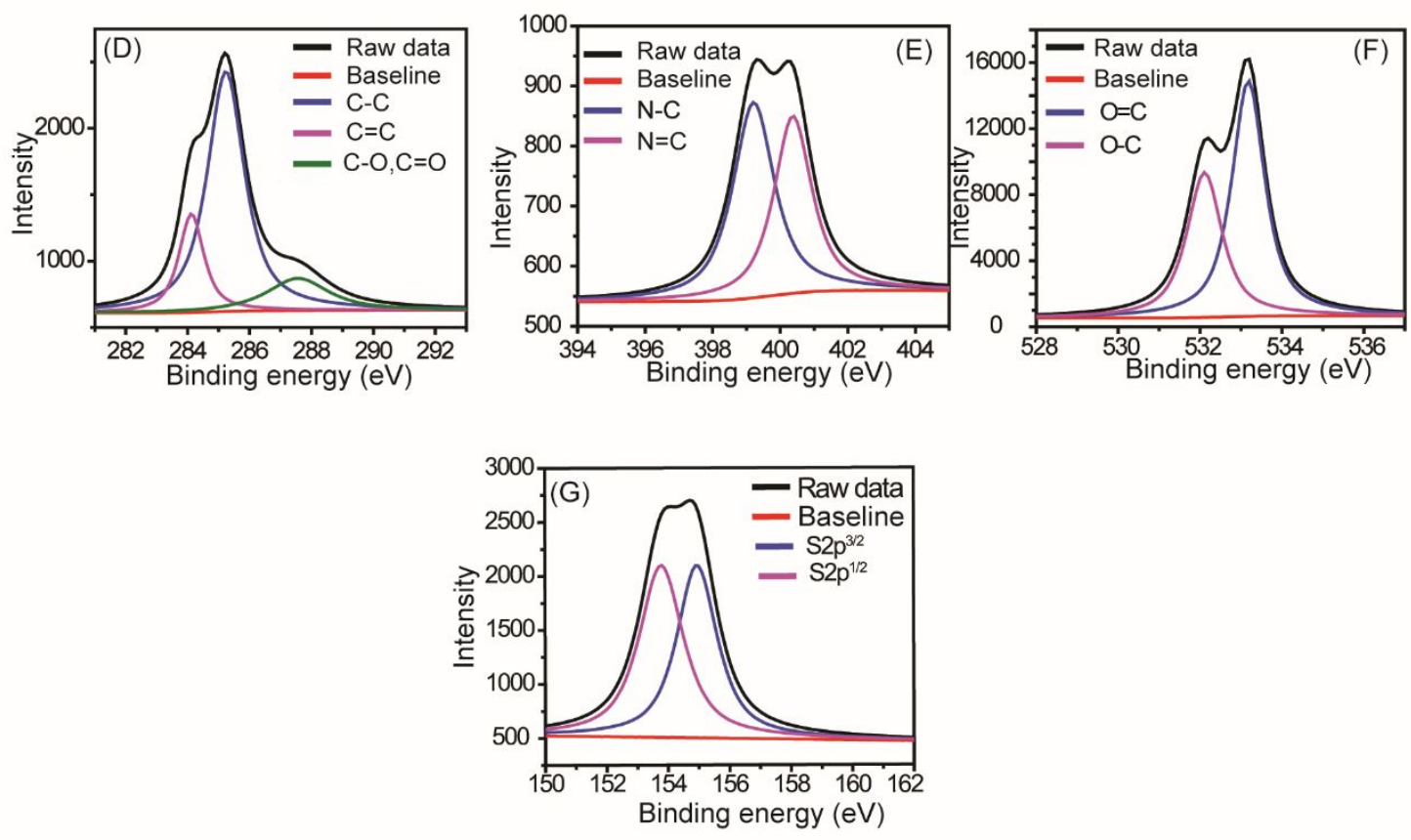

Figure S10. High resolution deconvoluting XPS Spectrum (D) C1s (E) N1s (F) O1s and (G) S2p of Cys C-Dots with black line (Raw data) and red line (baseline).
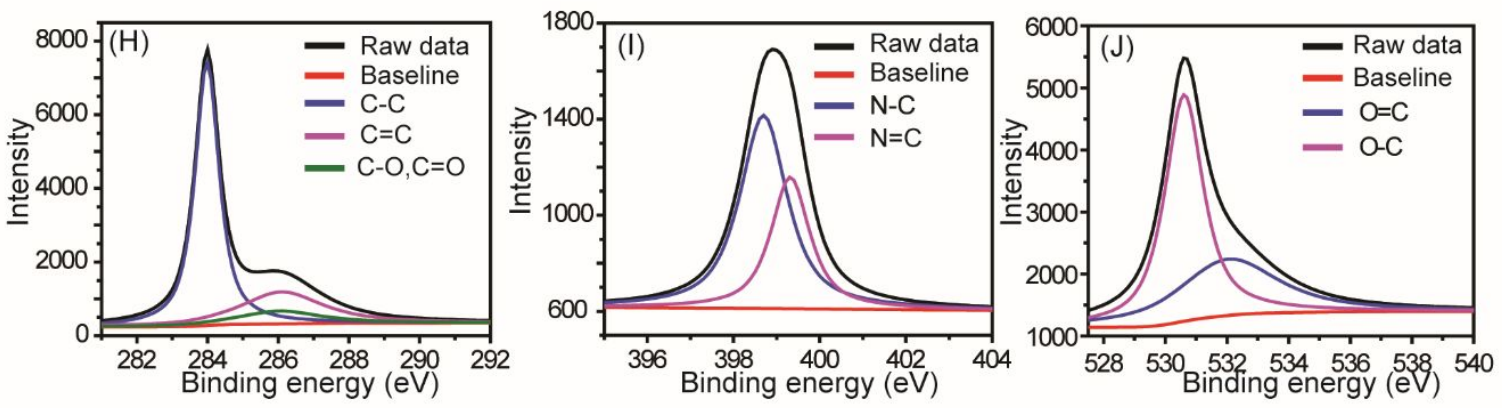
Figure S11. High resolution deconvoluting XPS Spectrum (H) C1s (I) N1s and (J) O1s of Leu CDots with black line (Raw data) and red line (baseline).

Figure S12. High Resolution deconvoluting XPS Spectrum (K) C1s (L) N1s and (M) O1s of His C-Dots with black line (Raw data) and red line (baseline).
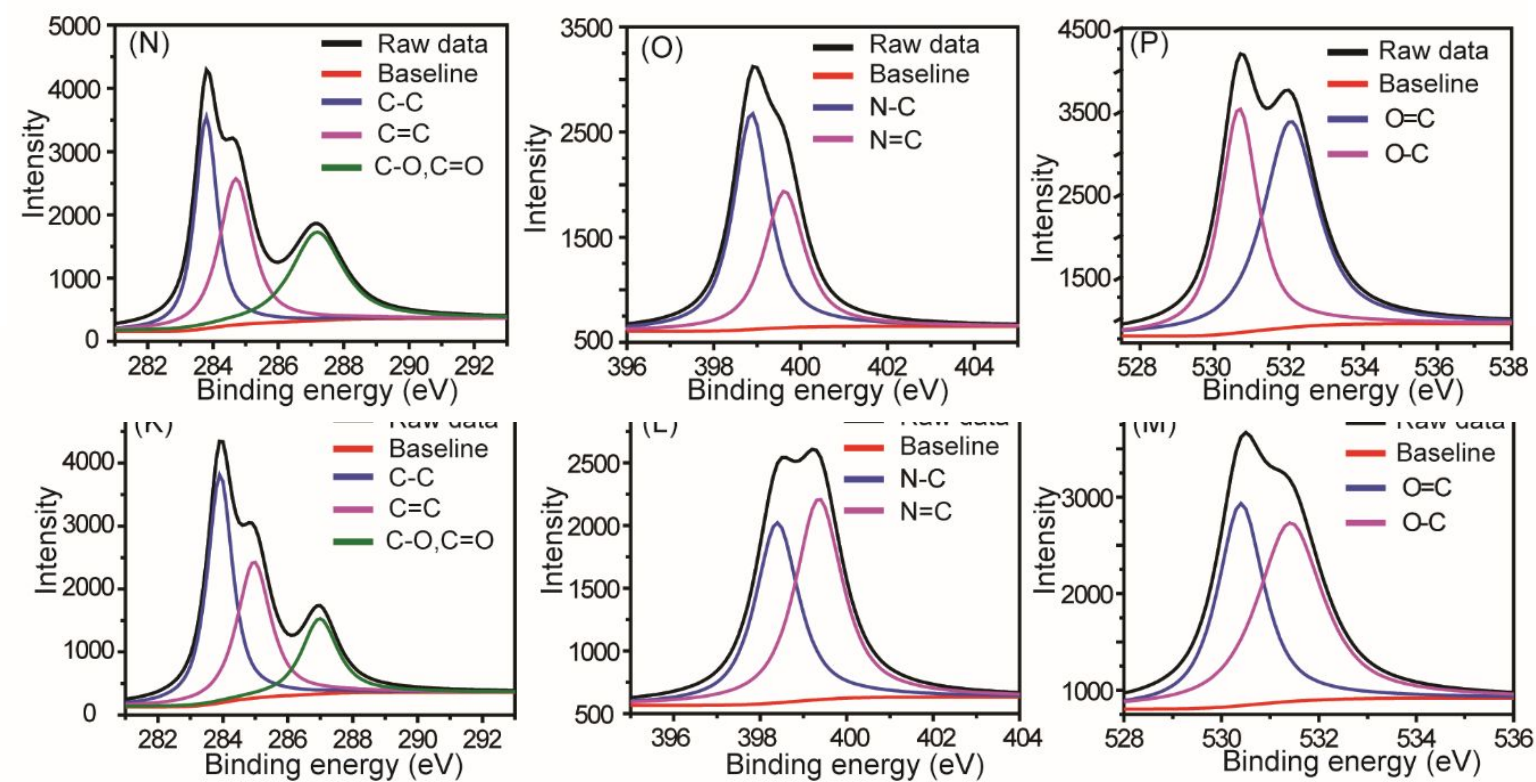

Figure S13. High resolution deconvoluting XPS Spectrum (N) C1s (O) N1s and (P) O1s of Phe C-Dots with black line (Raw data) and red line (baseline).

Table S1. Percentage of elements present in different amino acids functionalized C-Dots.

pXRD Study:

\begin{tabular}{|c|c|r|r|c|}
\hline Elements (\%) & $\mathrm{C}$ & $\mathrm{N}$ & $\mathrm{O}$ & $\mathrm{S}$ \\
\hline Arg & 68.56 & 17.37 & 14.07 & 0 \\
\hline Trp & 69.96 & 14.43 & 15.61 & 0 \\
\hline His & 73.71 & 14.21 & 12.08 & 0 \\
\hline Cys & 30.04 & 9.54 & 54.87 & 5.55 \\
\hline Glu & 71.98 & 13.33 & 14.69 & 0 \\
\hline Leu & 75.04 & 8.16 & 16.79 & 0 \\
\hline
\end{tabular}

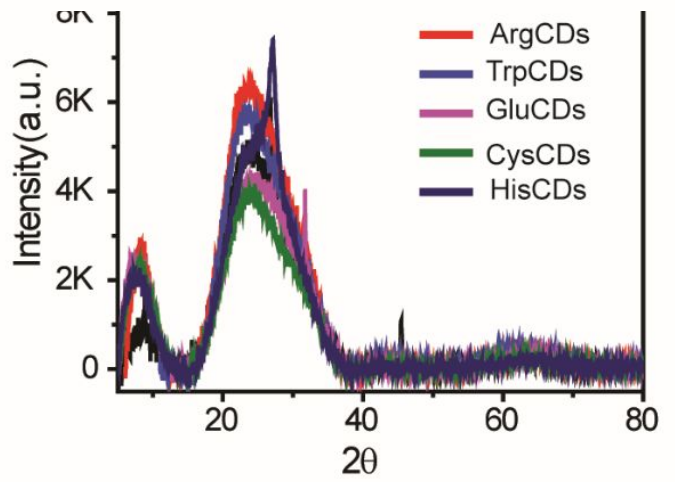


Figure S14. Powder X-Ray Diffraction (pXRD) of amino acids functionalized carbon dots Leu CDots (black), Arg C-Dots (red),Trp C-Dots (blue), Glu C-Dots (pink), Cys C-Dots (green) and His C-Dots (navy). 


\section{Fluorescence Spectra:}
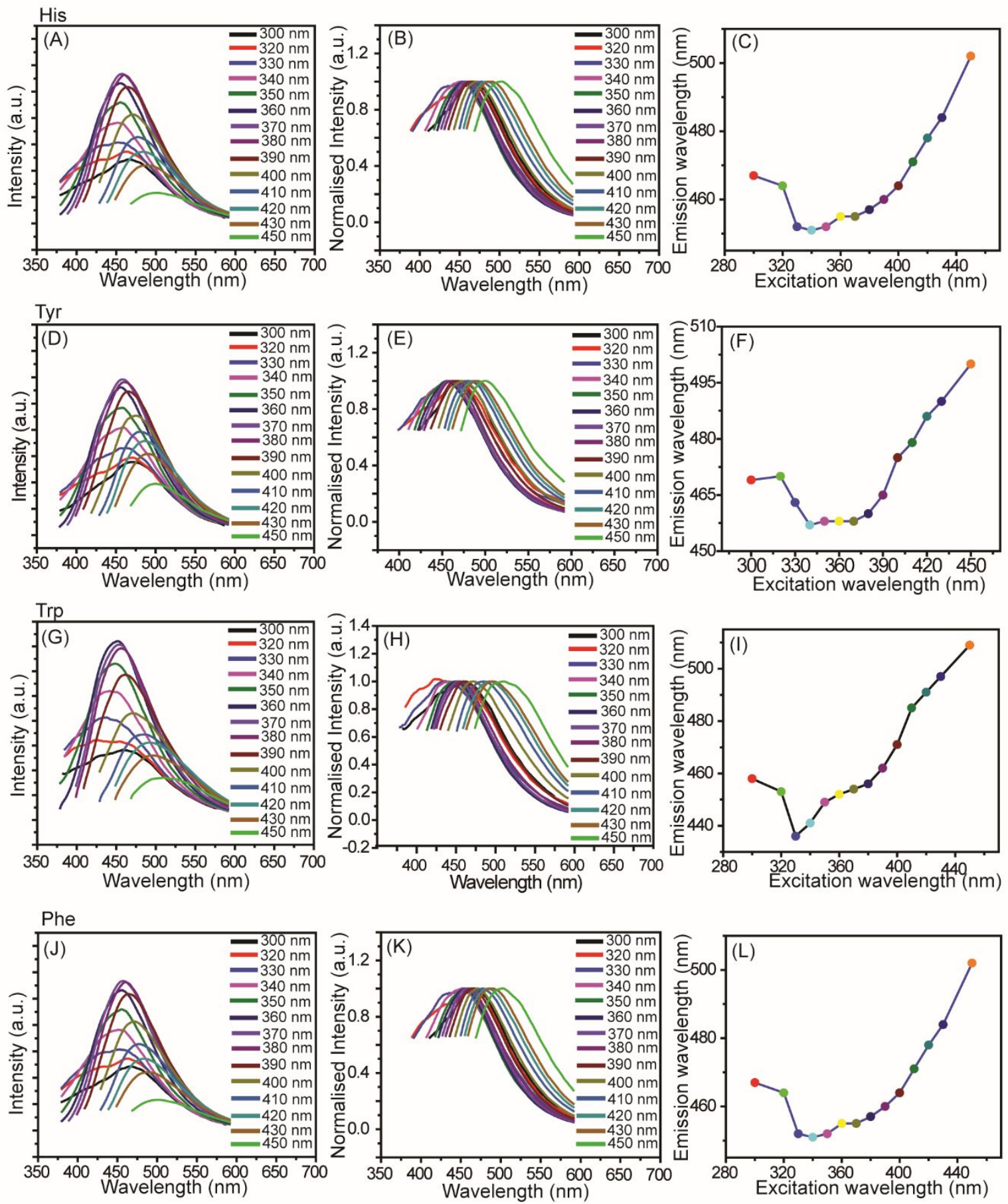

Figure S15. Fluorescence Spectra of all aromatic moiety amino acids functionalized carbon dots different excitation dependent fluorescence spectra, normalized spectra and excitation dependent emission spectra (A, B and C) for His C-Dots, (D, E, and F) for Tyr C-Dots, (G, H and I) for Trp C-Dots, (J, K and L) for Phe C-Dots respectively. 

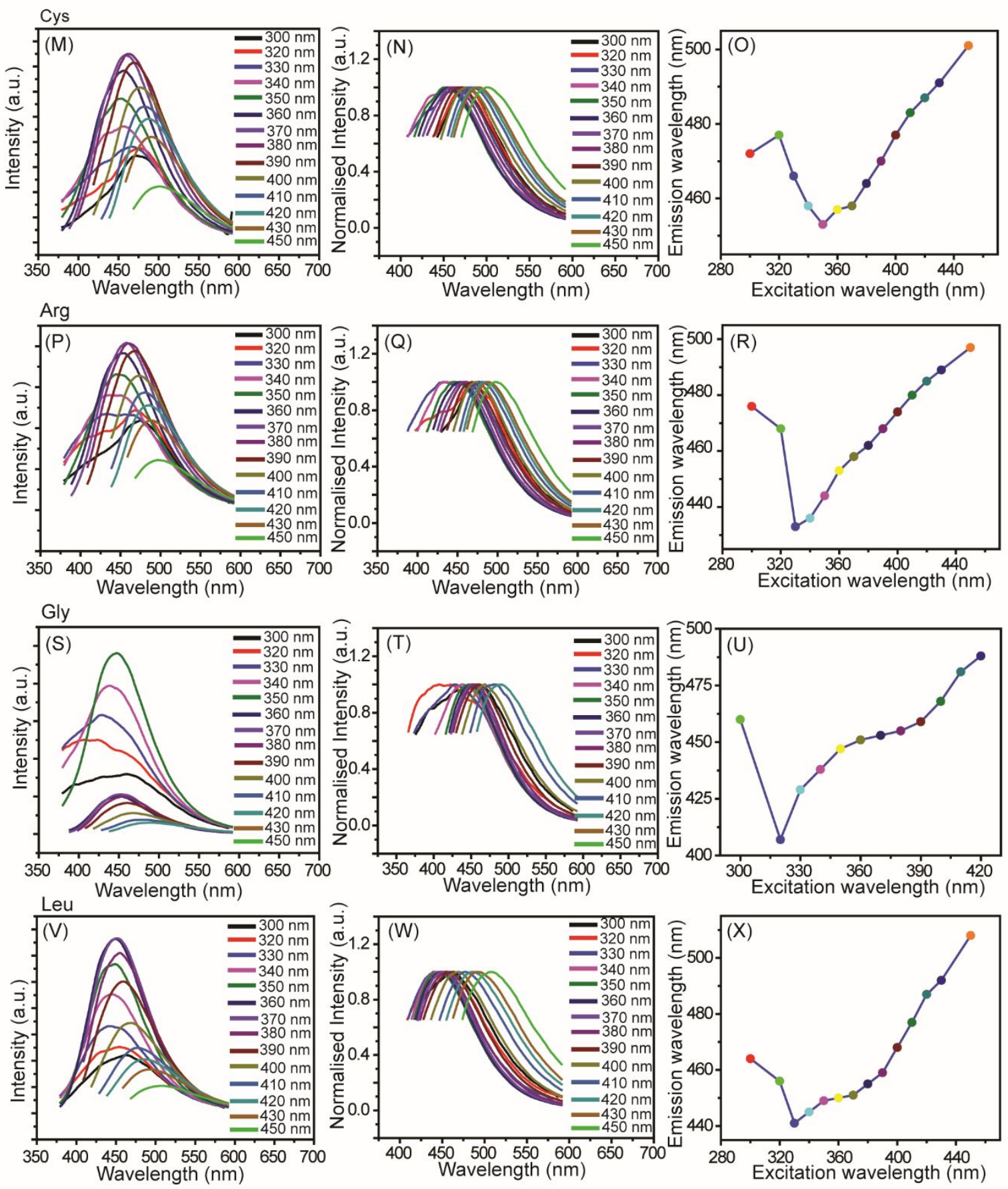

Figure S16. Fluorescence Spectra of side chain moiety amino acids functionalized carbon dots different excitation dependent fluorescence spectra, normalized spectra and excitation dependent emission spectra (M, N and O) for Cys C-Dots, (P, Q, and R) for Arg C-Dots, (S, T and U) for Gly C-Dots, (V, W and $\mathrm{X})$ for Leu C-Dots respectively. 

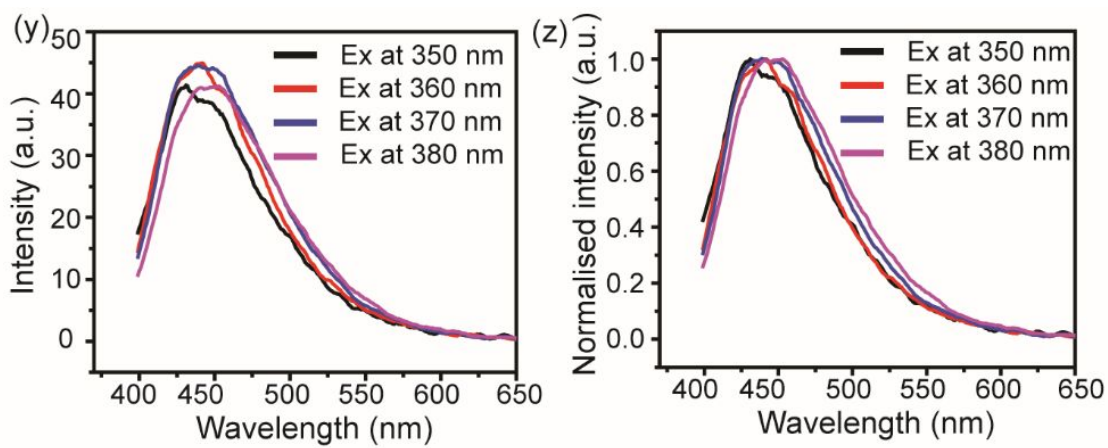

Figure S17. Fluorescence Spectra of only Citric acid C-Dots with excitation dependent fluorescence spectra(y); normalized spectra $(\mathrm{z})$ at identical conditions.

pH Study:
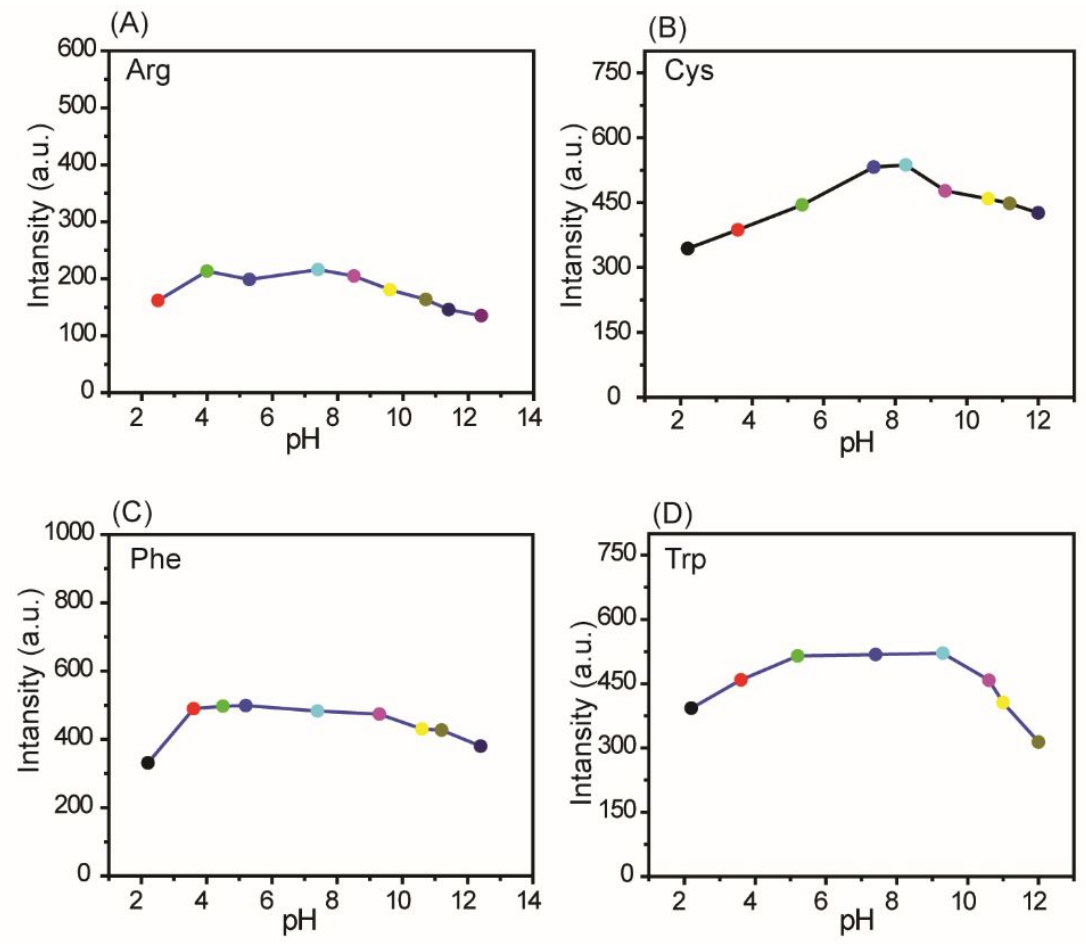

Figure S18. $\mathrm{pH}$ Stability of amino acids functionalized carbon dots (A) Arg C-Dots, (B) Cys CDots, (C) Phe C-Dots and (D) Trp C-Dots at pH range 2.2 to 12.5. 
Cellular cytotoxicity and targeted nucleolus tracking study:

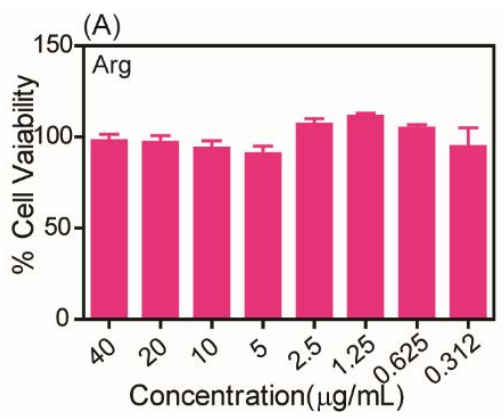

(D)

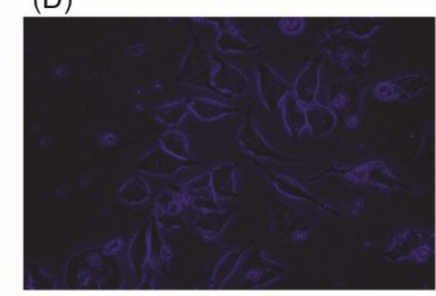

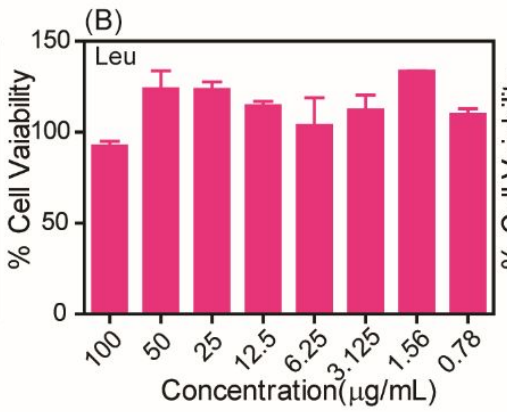

(E)

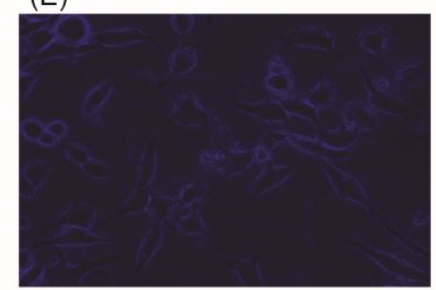

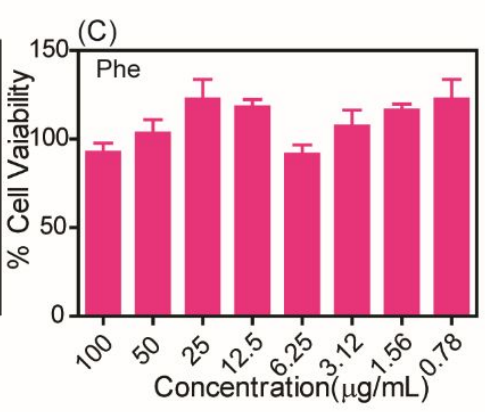

(F)

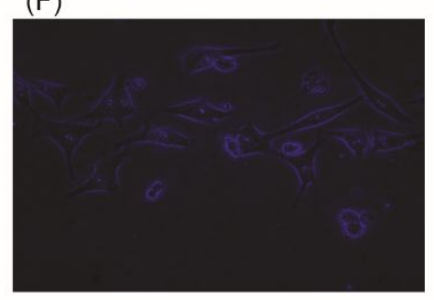

Figure S19. HeLa Cell viability study and targeted nucleolus tracking image of amino acids functionalized carbon dots (A, D) Arg C-Dots, (B, E) Leu C-Dots and (C, F) Phe C-Dots respectively.

Table S2. Five different proteins with diverse molecular weight and charge were chosen for the sensing experiment.

\begin{tabular}{|c|c|c|c|}
\hline Protein names & $\begin{array}{c}\text { Molecular weight in } \\
\text { kDa. }\end{array}$ & pI & $\begin{array}{c}\text { Molar Ex. } \\
\text { Coefficient }\end{array}$ \\
\hline CHYMOTRYPSIN & 25 & 8.7 & 51000 \\
\hline CYTOCHROME C & 12.3 & 10.7 & 23200 \\
\hline LIPASE & 58 & 5.6 & 54350 \\
\hline XYLANASE & 23.6 & 3.8 & 68000 \\
\hline BETA & 179.3 & 5.42 & 294930 \\
GLUCANASE & & & \\
\hline
\end{tabular}

